\title{
Disruptive Body Patterning of Cuttlefish (Sepia officinalis) Requires Visual Information Regarding Edges and Contrast of Objects in Natural Substrate Backgrounds
}

\author{
CHUAN-CHIN CHIAO ${ }^{1,2}$, EMMA J. KELMAN ${ }^{1,3}$, AND ROGER T. HANLON ${ }^{1, *}$ \\ ${ }^{1}$ Marine Biological Laboratory, Woods Hole, Massachusetts; ${ }^{2}$ Department of Life Science, \\ National Tsing Hua University, Hsinchu, Taiwan; and ${ }^{3}$ College of Life Sciences, University of Sussex, \\ Brighton, UK
}

Cuttlefish (Sepia officinalis Linnaeus, 1758) on mixed light and dark gravel show disruptive body patterns for camouflage. This response is evoked when the size of the gravel is equivalent to the area of the "White square," a component of its dorsal mantle patterns. However, the features of natural substrates that cuttlefish cue on visually are largely unknown. Therefore, we aimed to identify those visual features of background objects that are required to evoke disruptive coloration. At first, we put young cuttlefish in a circular experimental arena, presented them with natural gravel and photographs of natural gravel, and established that the animals would show a disruptive pattern when presented either with three-dimensional natural gravel or its two-dimensional photographic representation. We then manipulated the digital photographs by applying (i) a low-pass filter to remove the edges of the fragments of gravel, and (ii) a high-pass filter to remove the contrast among them. The body patterns produced by the cuttlefish in response to these altered visual stimuli were then videorecorded and graded. The results show that, to evoke disruptive coloration in cuttlefish, visual information about the edges and contrast of objects within natural substrate backgrounds is required.

Cephalopods have a remarkable ability to change the color and pattern of their skin, and research has demonstrated that visual input regulates these changes. Cuttlefish skin can show 20-50 chromatophore patterns that are used

Received 2 September 2004; accepted 10 December 2004.

* To whom correspondence should be directed. E-mail: rhanlon@ mbl.edu for both camouflage and communication (1). Cuttlefish can change their body patterns within a fraction of a second because chromatophore organs are innervated directly from the brain $(2,3)$. Because of its speed and diversity, body patterning in cuttlefish is the most sophisticated form of adaptive coloration in the animal kingdom (4). Although many aspects of cephalopod vision are known (5), the visual features of a given substrate that evoke adaptive coloration are relatively unstudied.

Recently we developed a quantifiable behavioral assay based upon single, static, computer-generated images that allow us to control detailed aspects of visual input. With this method, we first showed that certain visual background features were used by cuttlefish to produce disruptive coloration (6). Specifically, when the size of white squares on a checkerboard was similar to that of the "White square" component in the animal's skin, the cuttlefish produced a disruptive color pattern; this response occurred over a large contrast range and required only that a few white checks be present in the visual background. A subsequent study (7) showed that, to produce disruptive body patterns for camouflage, cuttlefish cue visually on the area - not the shape or aspect ratio- of light objects in a dark substrate. Most recently, we found that if the background was composed of a high density of small light and dark objects, the cuttlefish would produce mottled skin patterns; but if the background was uniform, uniformly stippled skin patterns would be produced (8). We also applied this behavioral assay to the study of the polarization vision of cuttlefish; although the results were mixed, they indicated that cuttlefish perceive differently polarized checks as light or dark objects and 
usually generate disruptive body patterns in response. The perceptual mechanisms underlying this polarization sensitivity are still unclear (9).

Taken together, this series of studies has demonstrated the utility of a laboratory sensorimotor assay in which sensory input can be measured quantitatively and its finetuned motor output (i.e., chromatophore skin patterns) can be easily seen and measured quantitatively in an intact, behaving cuttlefish (see also 10). This approach was first developed to study changeable coloration in flatfish $(11,12,13)$.

In these earlier investigations, cuttlefish were exposed to artificial—although readily quantified — substrates. We now examine the responses of cuttlefish to natural substrates and a variety of computer-manipulated digital photographs of those natural substrates. To achieve camouflage, animals must match various aspects of light and pattern. Thus, we expect that cuttlefish are attuned to certain elements of the spatial information in the background. Spatial frequency may be defined generally as the number of regular cycles in perceived light intensity per unit distance. The edge of an object, for example, might be characterized by a sharp transition in intensity, which is due to higher spatial frequencies of reflected light. In this paper, we show that for cuttlefish to detect light objects on a dark background and then produce disruptive body patterns, they require spatial frequency information about both the edges and contrast of background objects.

Four young cuttlefish, Sepia officinalis $(5-8 \mathrm{~cm}$ mantle length), were reared from eggs in the MBL Marine Resources Center (Woods Hole, MA) and were maintained in the same facility throughout these experiments. Each animal was placed in a tank $(30 \mathrm{~cm} \times 50 \mathrm{~cm} \times 15 \mathrm{~cm})$ with running seawater and was restricted by a circular arena $(25$ $\mathrm{cm}$ in diameter, $12 \mathrm{~cm}$ in height; walls were black) in which natural gravel and digital photographs of gravel backgrounds (laminated to be waterproof) were presented as the substratum. Natural gravel, collected at the local beach, was
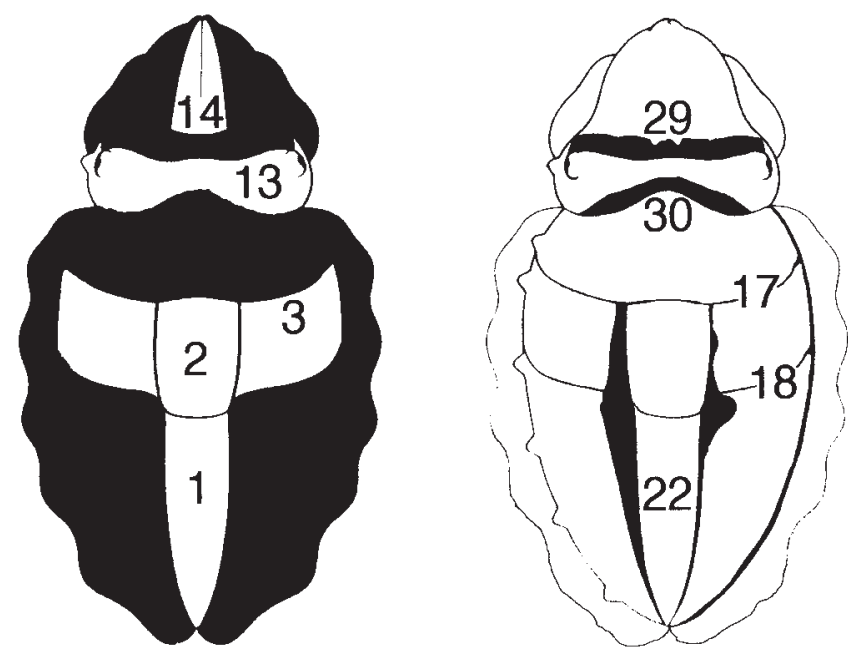

Figure 1. Ten chromatic skin components were used in grading the disruptive body patterns of the cuttlefish. The numbers of the components are the same as those used in reference 1. Five light components (left drawing) are numbered: (1) White posterior triangle; (2) White square; (3) White mantle bar, which includes White square and extends the full width of the mantle; (13) White head bar; and (14) White arm triangle. Five dark components (right drawing) are numbered: (17) Anterior transverse mantle line; (18) Posterior transverse mantle line; (22) Median mantle stripes (one on either side of the midline); (29) Anterior head bar; and (30) Posterior head bar. See text for grading method.

selected to be about the size of the White square component shown on the animal's dorsal mantle (illustrated as \#2 in Fig. 1). All of the gravel was glued, with standard acid-free rubber cement, onto sheets of acrylic plastic $(25 \mathrm{~cm} \times 25$ $\mathrm{cm} \times 0.5 \mathrm{~cm}$ ); thus the identical pattern of substrate could be presented in each trial. A digital picture of the glued gravel was taken with a Nikon Coolpix 5400 camera (5 megapixel image), and subsequent filtering and image enhancement were done in Photoshop CS (Adobe Systems, Inc.). A digital video camera (Sony VX-1000) was used to record the body patterning of S. officinalis over a period of $30 \mathrm{~min}$. The camera was set to record for $2 \mathrm{~s}$ every minute,

Figure 2. A-F: Responses of cuttlefish to a natural gravel substrate and to digital photographs of that identical substrate. (A) Cuttlefish (bottom right at 4 o'clock; near white mark in margin) shows a disruptive body pattern for camouflage on a natural gravel background. (B) Cuttlefish shows similar disruptive body pattern on a digital photograph of the gravel shown in (A). Low-pass (C) and high-pass (D) filtered images of the photograph shown in (B). On both backgrounds, the cuttlefish shows uniform or stippled patterns. The background shown in (E) is an enhanced contrast image of $(C)$, and $(F)$ is an enhanced contrast image of (D). In both, the body patterns of cuttlefish are similar to the original low-pass and high-pass filtered images (C and $\mathrm{D}$, respectively). Note, however, that the animal in (F) shows weak traces of the disruptive chromatic components White head bar, White square, and White posterior triangle. G-M: Statistical properties of processed images used in this study. The two-dimensional Fourier transform of the original digital image (G), the low-pass (H) and high-pass (I) filtering. The profiles of brightness distribution: the low-pass filtered image (J), the high-pass filtered image (K), the low-pass (L) and the high-pass (M) after Histogram Equalization. The gray scale $(0-255)$ is shown on the abscissa, and the number of pixels of each brightness level is indicated on the ordinate. Artifacts (i.e., two valleys form a sharp peak in the middle) can be seen in the Equalized high-pass filtered image $(\mathrm{M})$ due to the obscured brightness distribution of the high-pass filtered image (K), yet the overall contrasts of these filtered images were enhanced substantially (i.e., Fig. E and F). 

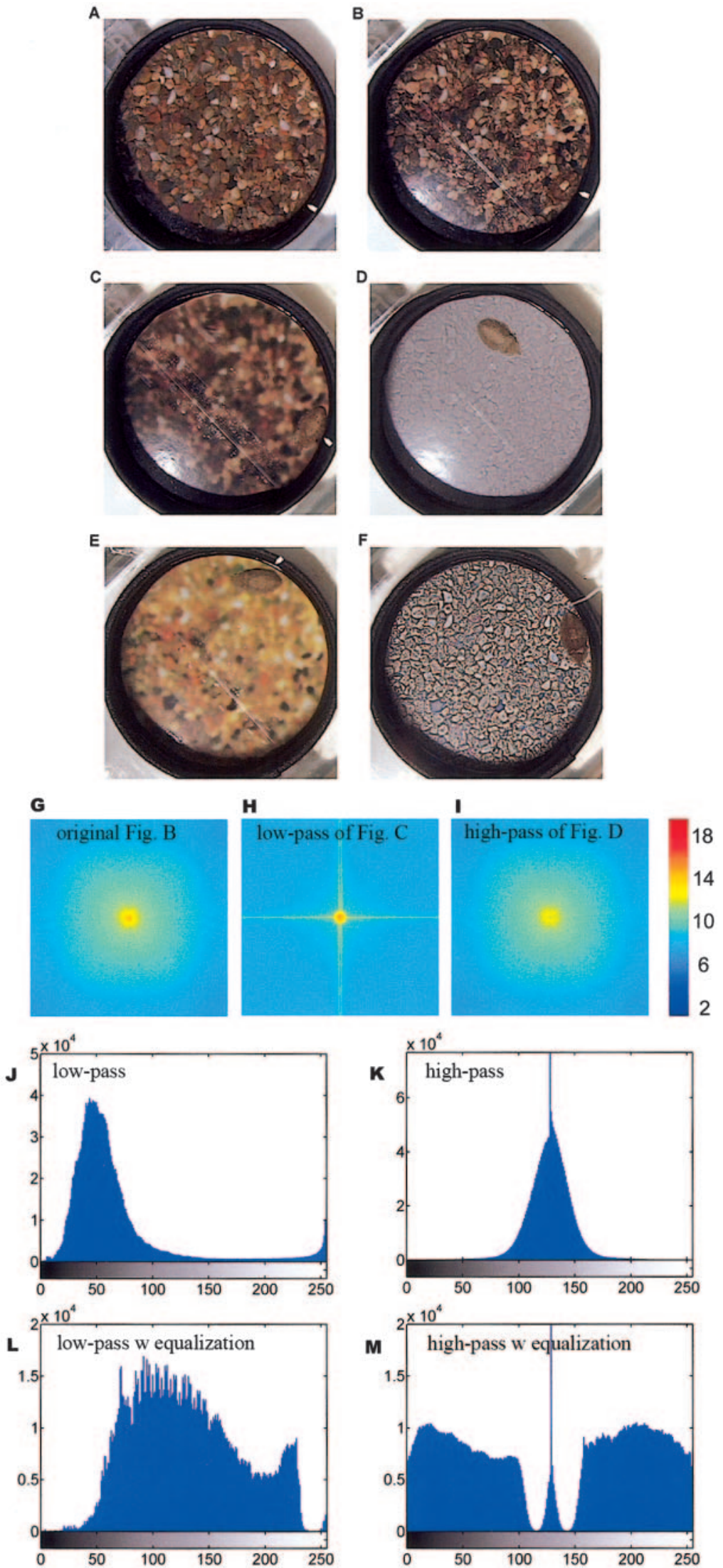
giving a total recording time of $60 \mathrm{~s}$ per animal per substrate. A 500-W tungsten light was used in all experiments. In earlier experiments $(6,7,8,9)$, the cuttlefish took several minutes to settle down on the black-and-white checkerboard substratum. In the present experiments, cuttlefish easily settled, not only on natural gravel, but also on the pictures of gravel.

To determine the responses of the animals to different substrata, a more precise grading scheme was applied to patterning of the skin $(6,7)$. In this refined grading scheme, five light and five dark disruptive chromatic components of the skin of head and mantle were evaluated and scored (1). The components are diagrammed in Figure 1, but also note the natural disruptive body patterns in Figure 2A, B. The following grades were assigned to each component: 2 , strong presence; 1 , partial presence; and 0 , absence. Partial or strong presence was determined by the relative degree of contrast between the light and dark components of the skin. Grading was conducted by playing the videotape and assigning a grade (whole integers $0,1,2$ ) every $6 \mathrm{~s}$ for each component on the skin. Thus, since all tapes lasted $60 \mathrm{~s}$, the components on each animal were graded 10 times on each substratum. Each disruptive chromatic component was graded separately and with equal weight, and grades for all components were summed to yield the final scores. For example, a score of 20 represents the maximal disruptive appearance: strong presence (grade 2) of all 10 disruptive components. A score of zero represents the minimal disruptive appearance: uniform or stippled patterns. Scores of 8-15 represent typical disruptive patterns, as described often $(1,2,4,6,7,9)$.

Figure 2 illustrates various manipulations of the substrate image. First, we removed the high spatial frequency information from the scene (i.e, the edges of objects in the substrate). In brief, we used Photoshop to apply a low-pass (or Gaussian) blur filter to the original image, which was 900 pixels in diameter. The Gaussian filter had a radius of 10 pixels and a cutoff frequency at $1.3 \%$ of the Nyquist frequency of the image (14). Second, we removed low spatial frequency information from the substrate image (i.e., local contrast between objects in the substrate) by applying a high-pass filter in Photoshop (14); this filter had a 10-pixel radius and a cutoff frequency at $98 \%$ of the Nyquist frequency of the image. Thus, the effects of the Gaussian filter (Fig. 2C) and the high-pass filter (Fig. 2D) are opposed. Two-dimensional Fourier transforms of the original image and the low-pass and high-pass-filtered images are compared in Figure 2G, H, and I. The rationale for using Fourier transformations to analyze spatial frequency informationand the methodology for carrying the analysis out-are provided elsewhere (15). Since images tend to lose overall contrast due to filtering effects, we applied a maximal contrast-enhancing tool to boost the contrast of the filtered images. The Equalize function in Photoshop was used; this is identical to Histogram Equalization, where a brightness distribution is normalized so that all values are equally probable. The profiles of the brightness distribution of the low-pass and the high-pass filtered images, before and after maximal contrast enhancement (Histogram Equalization), are shown in Figure 2J-M. Note that Histogram Equalization can only approximate the equally probable intensity values for these images.

Cuttlefish showed strongly disruptive patterns on natural gravel (Fig. 2A), as expected from previous results $(1,2)$. More important, cuttlefish also showed similarly disruptive patterns on the exact photograph of natural gravel (Fig. 2B). This indicates that cuttlefish use mainly visual cues for camouflage; i.e., a tactile cue is not required (1). These results imply further that cuttlefish can sense the essential visual cues for disruptive camouflage from two-dimensional images, and that the three-dimensional cues are less vital for this particular task.

After establishing that animals responded similarly to three-dimensional natural gravel and two-dimensional gravel pictures, we sought to examine those gross aspects of spatial frequency in the scene that might contain critical visual cues informing the cuttlefish's choice of camouflage patterning. From earlier experiments (7), we knew that to produce disruptive body patterns, cuttlefish cue visually on the area-not shape or aspect ratio- of white objects on a dark background. This suggested that cuttlefish may determine the size of white objects by visually integrating the areas of whiteness. But whether cuttlefish use the edges or the local contrast between white objects and the dark background (or both) to make this determination is not known.

Cuttlefish did not show disruptive colorations on either the low-pass (Fig. 2C) or the high-pass (Fig. 2D) filtered images. Furthermore, cuttlefish did not respond to the filtered images of enhanced overall contrast (Fig. 2E, F). Although only two animals were tested, this result suggests that cuttlefish require both edges and local contrast to recognize the objects, and that global-contrast-enhanced images have little effect on their patterning. Responses from four animals are summarized in Figure 3. There was no significant difference (Student's $t$ test) between the animals' responses on natural gravel and on the original digital photograph of gravel $(P>0.38)$. However, there were significant differences between the cuttlefishes' body patterns on the original digital photograph and on the various modified images $(P<0.01$, low-pass; $P<0.001$, enhanced low-pass; $P<0.001$, high-pass; $P<0.013$, enhanced high-pass).

The result, that animals responded differently on the original digital photograph of gravel and the blurred picture of gravel, supports the notion that the cuttlefish visual system has good spatial resolution (16), and that the details of the scene (e.g., the edges of objects) are important for cuttlefish to produce appropriate camouflage patterns. Ver- 


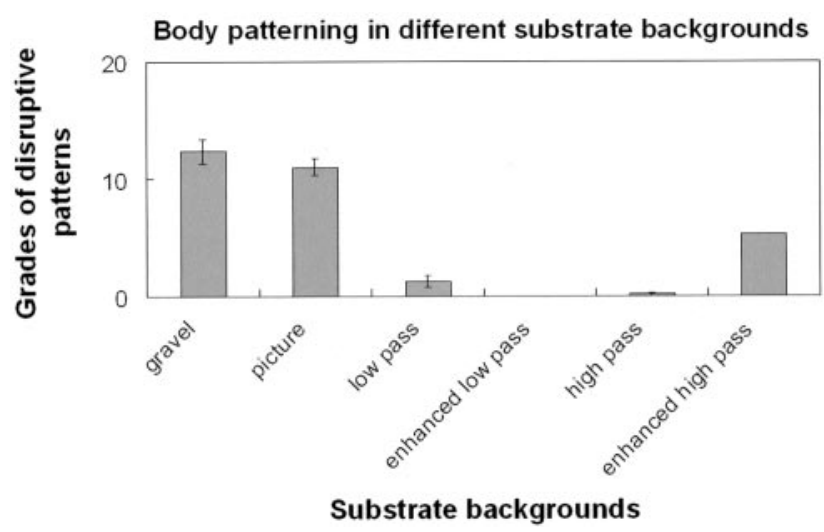

Figure 3. Grades of cuttlefish disruptive body patterning on natural gravel substrate and various digital photographs of natural gravel. Averaged grades of four cuttlefish on different substrata are shown, and bars represent the standard errors. For enhanced contrast images (enhanced low-pass and enhanced high-pass), only two animals were tested.

tebrate visual systems tend to use edge detection to recognize an object. In our experimental design, however, edge alone did not provide a sufficient cue for cuttlefish to produce disruptive coloration. Cuttlefish visually discern light objects (6), thus to show disruptive patterns for camouflage on the mixed light and dark natural gravel backgrounds (Fig. 2A), the local contrast between light and dark gravel is an essential visual cue. This finding is also consistent with our earlier results showing that reducing the contrast of black-and-white checkerboards affected the cuttlefishes' disruptive pattern (6), although in those trials only the stark black-and-white checkerboards were tested, whereas in the current trials, the gravel had many more shades of gray. Figure $2 \mathrm{~F}$ is interesting because the edges are discernible to human vision and contrast is noticeable; yet the cuttlefish responded with only a very weak White square, White head bar, and White posterior triangle, possibly indicating a threshold of edge detection.

Sepia officinalis lives in a variety of substrata and uses a complex repertoire of changeable body patterns for camouflage (1). Our results show that the type of patterning chosen for display in a cuttlefish's skin is based upon a wide range of visual information. Our study also provides insight into how cuttlefish extract visual information from natural substrates. However, the animals' responses on various filtered images of a natural substrate were not thoroughly examined in this somewhat preliminary study; nor were the quantitative characteristics of the animals' responses to edges and contrast determined. Future studies will take advantage of this noninvasive behavioral assay to examine these matters and other visual processing mechanisms of cuttlefish.

\section{Acknowledgments}

We appreciate valuable comments from Phil McFadden, Lydia Mathger, Eric Warrant, the editor, and two anonymous reviewers. We are grateful for funding from the Sholley Foundation and Anteon contract \#USAF-5408-04-SC0002. Alexandra Barbosa and staff of the Marine Resources Center provided important animal care.

\section{Literature Cited}

1. Hanlon, R. T., and J. B. Messenger. 1988. Adaptive coloration in young cuttlefish (Sepia officinalis L.): the morphology and development of body patterns and their relation to behaviour. Philos. Trans. R. Soc. Lond. B 320: 437-487.

2. Hanlon, R. T., and J. B. Messenger. 1996. Cephalopod Behaviour. Cambridge University Press, Cambridge.

3. Messenger, J. B. 2001. Cephalopod chromatophores: neurobiology and natural history. Biol. Rev. 76: 473-528.

4. Cott, H. B. 1940. Adaptive Coloration in Animals. Methuen, London.

5. Messenger, J. B. 1991. Photoreception and vision in molluscs. Pp. 364-397 in Evolution of the Eye and Visual System, J. R. Cronly-Dillon and R. L. Gregory, eds. Macmillan Press, London.

6. Chiao, C.-C., and R. T. Hanlon. 2001. Cuttlefish camouflage: visual perception of size, contrast and number of white squares on artificial checkerboard substrata initiates disruptive coloration. J. Exp. Biol. 204: 2119-2125.

7. Chiao, C.-C., and R. T. Hanlon. 2001. Cuttlefish cue visually on area-not shape or aspect ratio- of light objects in the substrate to produce disruptive body patterns for camouflage. Biol. Bull. 201: 269270 .

8. Barbosa, A., C. F. Florio, C.-C. Chiao, and R. T. Hanlon. 2004. Visual background features that elicit mottled body patterns in cuttlefish, Sepia officinalis. Biol. Bull. 207: 154 (Abstract).

9. Grable, M. M., N. Shashar, N. L. Gilles, C.-C. Chiao, and R. T. Hanlon. 2002. Cuttlefish body patterns as a behavioral assay to determine polarization perception. Biol. Bull. 203: 232-234.

10. Marshall, N. J., and J. B. Messenger. 1996. Colour-blind camouflage. Nature 382: 408-409.

11. Mast, S. O. 1914. Changes in shade, color, and pattern in fishes, and their bearing on the problems of adaptation and behavior, with especial reference to the flounders Paralichthys and Ancylopsetta. Bulletin of the Bureau of Fisheries, Wash. 34: 173-238.

12. Saidel, W. M. 1988. How to be unseen: an essay in obscurity. Pp. 487-513 in Sensory Biology of Aquatic Animals, R.R. Fay, A.N. Popper, and W.N. Tavolga, eds. Springer-Verlag, New York.

13. Ramachandran, V., C. W. Tyler, R. L. Gregory, D. Rogers Ramachandran, S. Duensing, C. Pillsbury, and C. Ramachandran. 1996. Rapid adaptive camouflage in tropical flounders. Nature 379: 815-818.

14. Russ, J. C. 1995. The Image Processing Handbook, 2nd ed. CRC Press, Boca Raton, FL.

15. Godfrey, D., J. N. Lythgoe, and D. A. Rumball. 1987. Zebra stripes and tiger stripes: The spatial frequency distribution of the pattern compared to that of the background is significant in display and crypsis. Biol. J. Linn. Soc. 32: 427-433.

16. Watanuki, N., G. Kawamura, S. Kaneuchi, and T. Iwashita. 2000. Role of vision in behavior, visual field, and visual acuity of cuttlefish Sepia esculenta. Fish. Sci. 66: 417-423. 